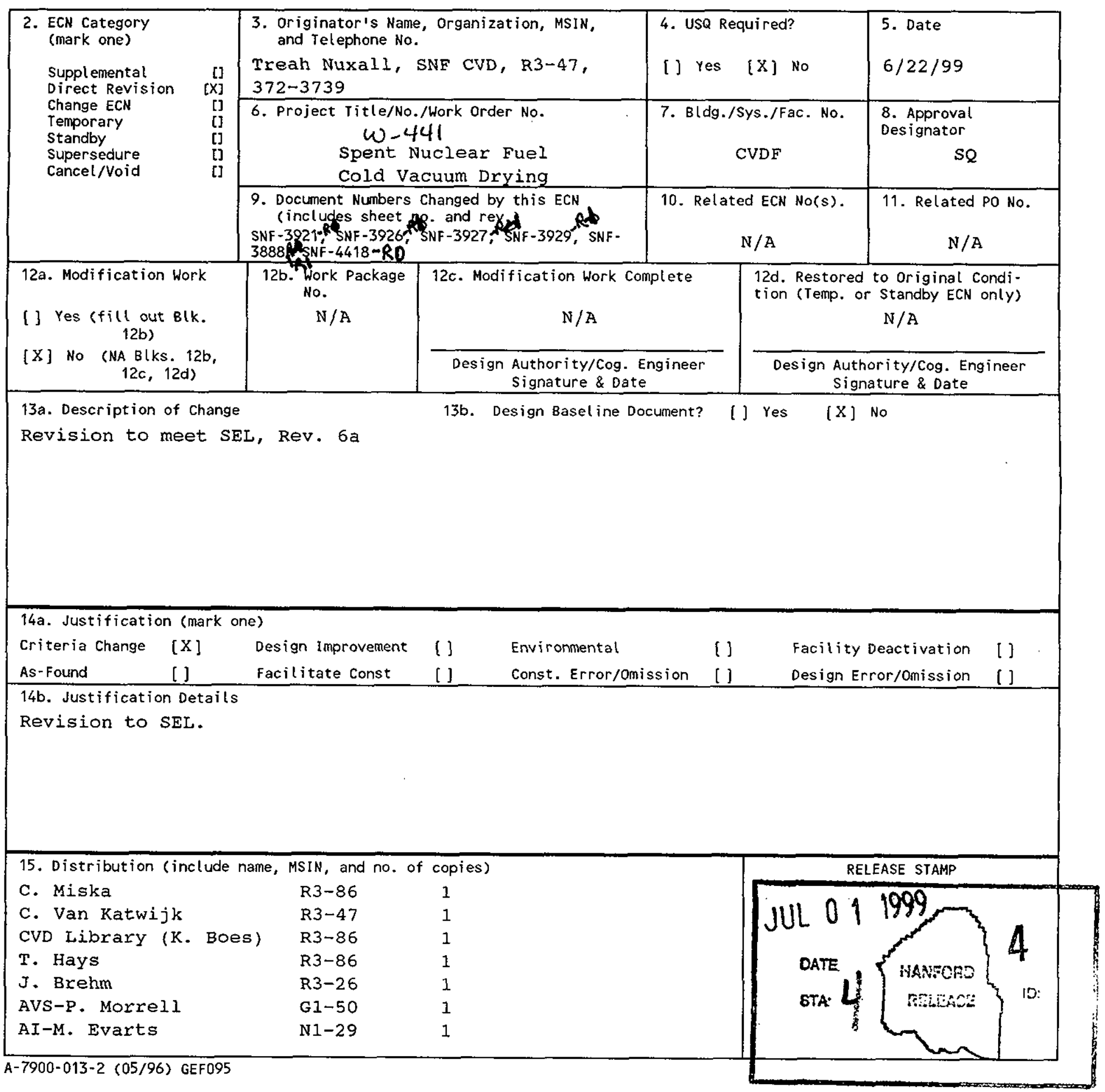




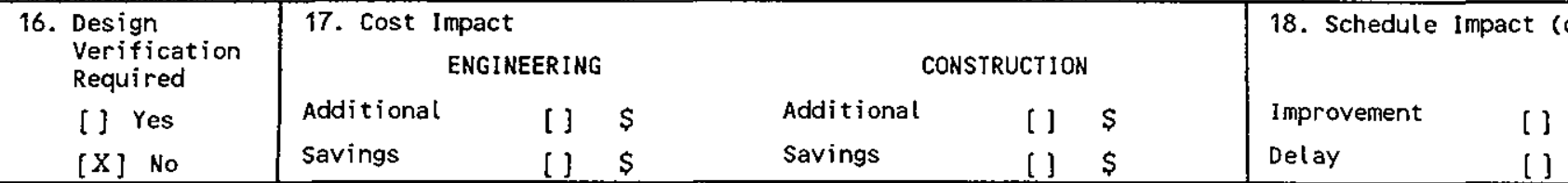

19. Change Impact Review: Indicate the related documents (other than the engineering documents identified on side 1 ) that will be affected by the change described in Block 13 . Enter the affected document number in Block 20.

$\begin{array}{ll}\text { SOD/DD } & {[} \\ \text { Functional Design Criteria } & {[]} \\ \text { Operating Specification } & {[]} \\ \text { Criticality Specification } & {[]} \\ \text { Conceptual Design Report } & {[]} \\ \text { Equipment Spec. } & {[]} \\ \text { Const. Spec. } & {[]} \\ \text { Procurement Spec. } & {[]} \\ \text { Vendor Information } & {[]} \\ \text { OM Manual } & {[]} \\ \text { FSAR/SAR } & {[]} \\ \text { Safety Equipment List } & {[]} \\ \text { Radiation Work Permit } & {[]} \\ \text { Environmental Impact Statement } & {[]} \\ \text { Environmental Report } & {[]} \\ \text { Environmental Permit } & {[}\end{array}$

[] Seismic/Stress Analysis

Stress/Design Report
Interface Control Drawing
Calibration Procedure
Installation Procedure
Maintenance Procedure
Engineering Procedure
Operating Instruction
Operating Procedure
Operational Safety Requirement
IEFD Drawing
Cell Arrangement Drawing
Essential Material Specification
Fac. Proc. Samp. Schedule

[]
[]

Process Control Manual/Plan

20. Other Affected Documents: (NOTE: Documents listed below will not be revised by this ECN.) Signatures below indicate that the signing organization has been notified of other affected documents listed below. Document Number/Revision Document Number/Revision Document Number Revision

N $/ A$

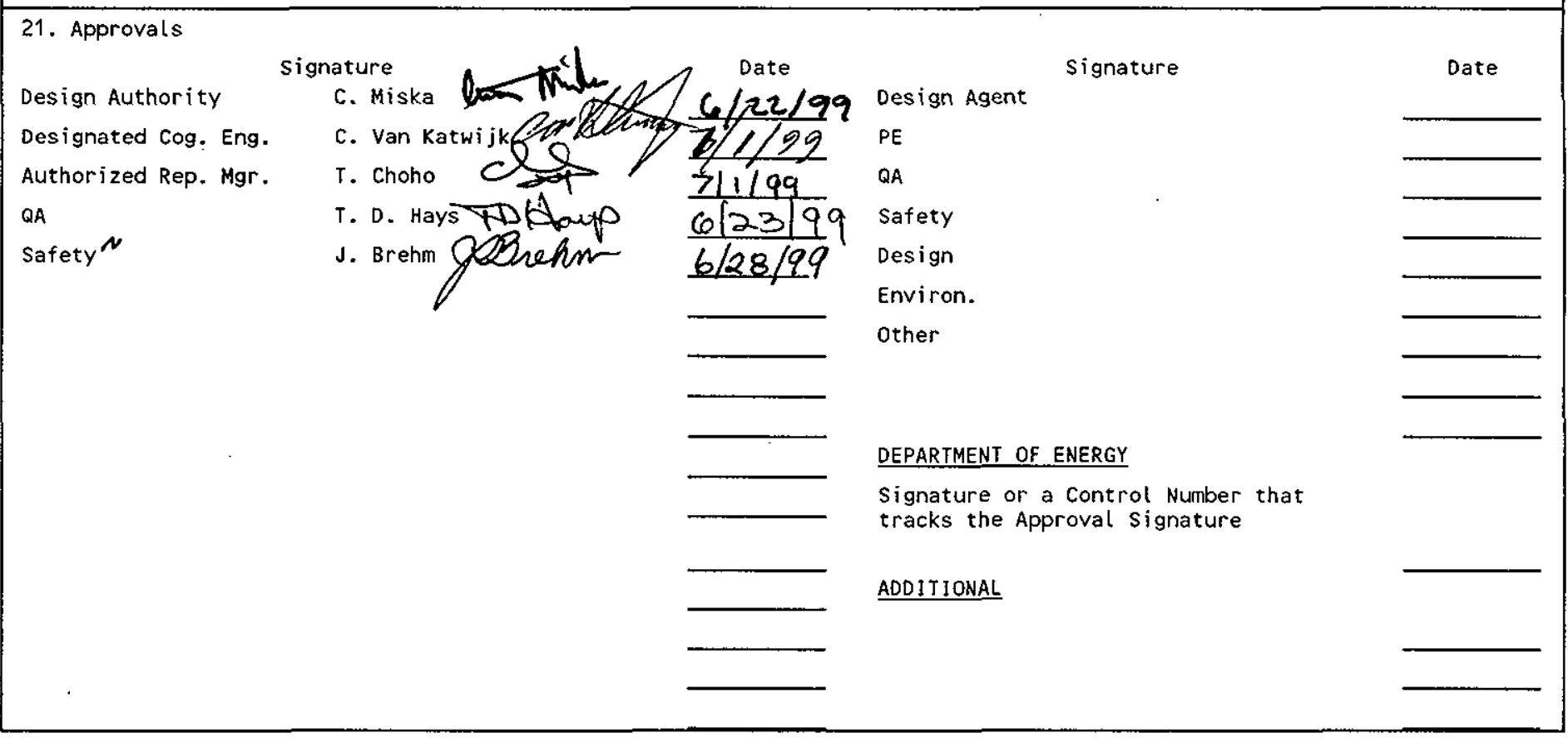




\section{MATHESON SCHe PRESSURE REGULATOR (SCHe TANK OUTLET)}

Carl Van Katwijk

Numatec Hanford, Richland, WA 99352

U.S. Department of Energy Contract DE-AC06-96RL13200

EDT/ECN: 647508

UC: 620

Org Code: $2 \mathrm{G} 300$

Charge Code: $105559 / \mathrm{A} 000$

B\&R Code: 39EW40400

Total Pages: 13

Key Words: Matheson SCHe Pressure Regulator

Abstract: Matheson SCHe Pressure Regulator

TRADEMARK DISCLAIMER. Reference herein to any specific commercial product, process, or service by trade name, trademark, manufacturer, or otherwise, does not necessarily constitute or imply its endorsement, recommendation, or favoring by the United States Government or any agency thereof or its contractors or subcontractors.

Printed in the United States of America. To obtain copies of this document, contact: Document Control Services, P.O. Box 950, Mailstop H6-08, Richland WA 99352, Phone (509) 372-2420; Fax (509) 376-4989.

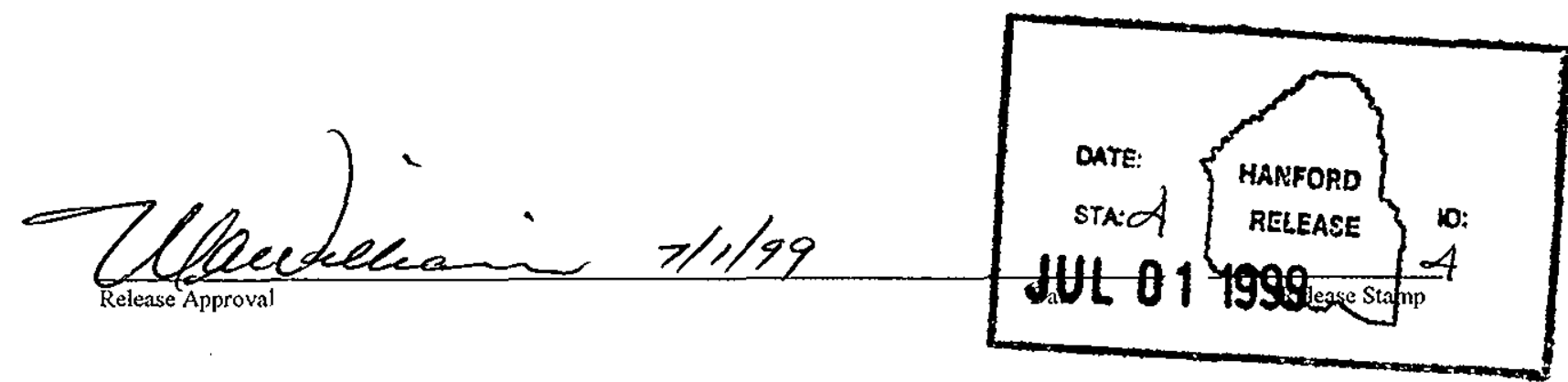

Approved for Public Release 
RECORD OF REVISION

(2) Title

Matheson SCHe Pressure Regulator (SCHe Tank Outlet)

Change Control Record

(3) Revision

2 Ps
(4) Description of Change - Replace, Add, and Delete Pages (7) ECN 647508, Revision to meet SEL Rev. 6a
Authorized for Release

\begin{tabular}{l|ll} 
(5) Cog. Engr. & (6) Cog. Mgr. Date
\end{tabular}

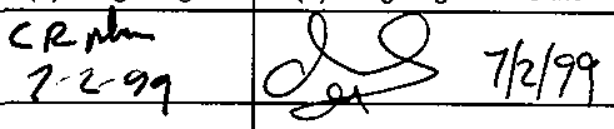


Commercial Grade Item Upgrade Dedication Form

ECN No. NA CGI No. CGI-SNF-D-13-P5-032

SNF-3929, Rev. 2

Title: MATHESON SCHe PRESSURE REGULATOR (SCHe TANK

OUTLET)

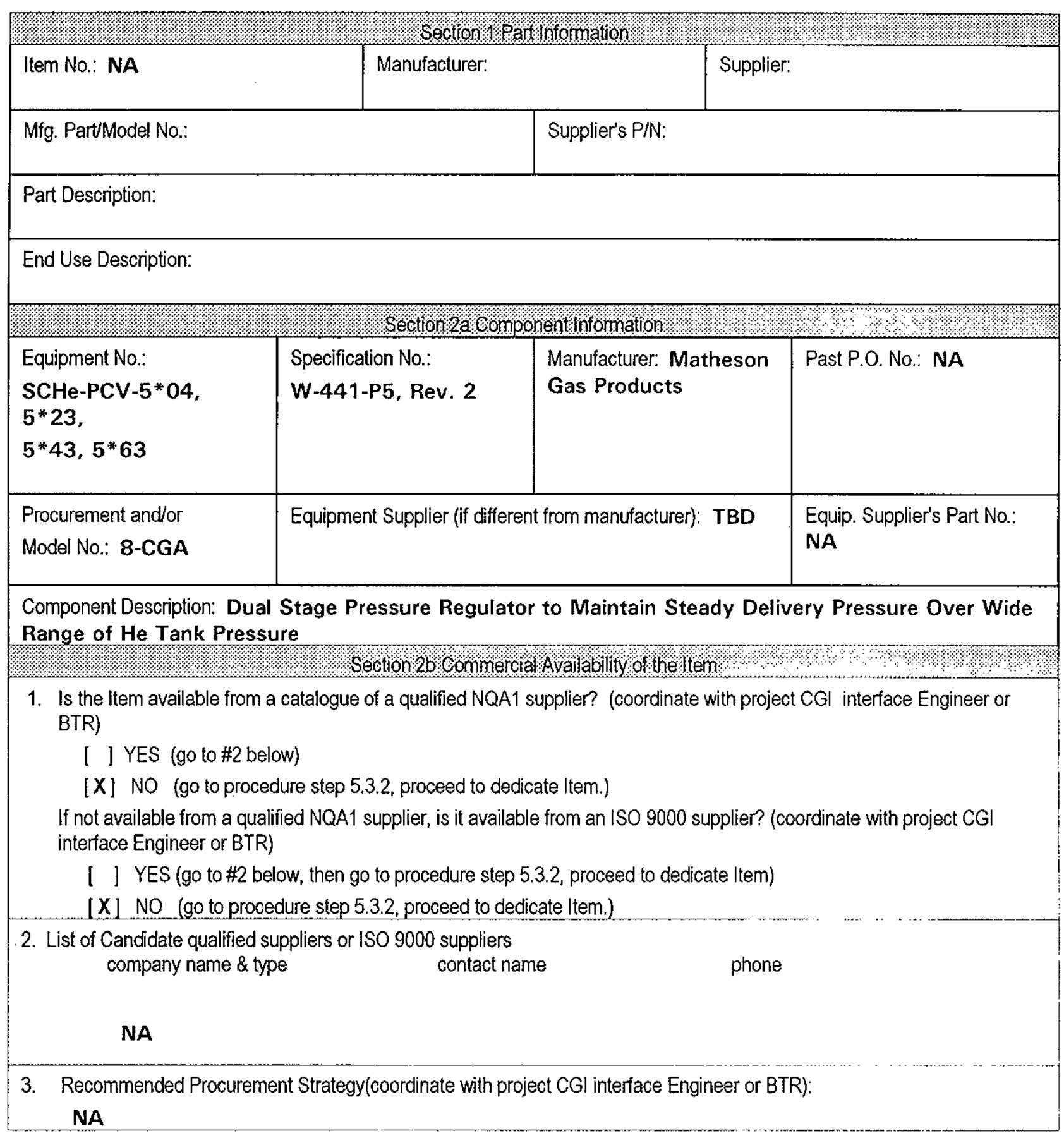




\section{Section 2c CGI Determination}

1. Question \#1: Is the Item subject to design or specification requirements that are unique to nuclear facilities or activities?

[ ] YES (the item is not commercial grade)

[X] NO (continue)

2. Question \#2: Is the Item used in applications other than nuclear facilities or activities?

[ ] NO (the item is not commercial grade)

[X] YES (continue)

3. Question \#3: Is the Item ordered from manufacturer/supplier on the basis or specifications set forth in the Published product information (e.g., manufacturer's catalog)?

[ ] NO (the Item is not commercial grade)

[X] YES (continue)

[X] All three criteria have been satisfied. The ltem meets the definition of commercial grade.

Secion 2 d Reason for bedication

The above descrbed then is being Bedicated for use th the application cited or the following reason(s):

[ $\mathbf{X}$ ] Item is being purchased from a non ESL manufacturer supplier as commercial grade to be used in a Safety Class application.

[ ] Item is being purchased from a non ESL manufacturer supplier as commercial grade to be used in a Safety Significant application.

[ ]. Item was purchased from a non ESL manufacturer supplier as commercial grade to be used in a Safety Class application.

[ ] Item was purchased from a non ESL manufacturer supplier as commercial grade to be used in a Safety Significant application.

L l Other ('like-for-like', similar, substitution, replacement evaluation)

Section 3 Eallure Effects Evaluation

A. Part/Component Safety Function:

1. Pressure boundary/confinement.

2. Prevent $\mathrm{H}_{2}$ explosion.

3. Maintain critical function before and after seismic event.

B. Part/Component Functional Mode:

Safety Function \#1:

l ] Active - Mechanical or Electrical change of state is required to occur for the component to perform its safety function

[X] Passive - Change of state is not required for the component to perform its safety function

Safety Function \#2:

[ ] Active - Mechanical or Electrical change of state is required to occur for the component to perform its safety function.

[X] Passive - Change of state is not required for the component to perform its safety function

Safety Function \#3:

1 ] Active - Mechanical or Electrical change of state is required to occur for the component to perform its safety function.

[X] Passive - Change of state is not required for the,component to perform its safety function

C. Host Component Safety Function (if applicable): NA

1. 
Commercial Grade Item Upgrade Dedication Form

ECN No. NA CGI No. CGI-SNF-D-13-P5-032

SNF-3929, Rev. 2

Title: MATHESON SCHE PRESSURE REGULATOR (SCHE TANK

OUTLET)

D. Failure Mode(s) and the effects on component or system safety function (see Worksheet 1):

1. Valve Body/Process Connection break - loss of boundary, air in-leakage.

2. Diaphragm/Body Failure - loss of regulating function.

section t/ Environmental \& Natural Phenomera a lazard Design

Environmental Qualification Required:

If yes: Environmental Qualification Requirements

Yes [ ]

Limiting Environmental Conditions:

No $[\mathbf{X}]$

Environmental Condition $\mathrm{B}$

Required Safety Functions:

Qualification Period:

Natural Phenomena Hazard (NPH) Design Required:

$$
\begin{aligned}
& \text { Yes [ } \mathbf{X}] \\
& \text { No [ ] }
\end{aligned}
$$

If yes: NPH Design Requirements

Performance Category: PC-3

NPH Design Req'ts.: Seismic Condition A

HNF-PRO-97, Rev. 0

Required Safety Functions: Pressure Boundary

W-441-P5, Rev. 2

\begin{tabular}{|c|c|}
\hline & Section 5 component r unctonalelassification \\
\hline$[\mathrm{X}]$ Safety Class $(\mathrm{SC})$ & [ ] General Service $\quad$ ] ] Safety Significant (SS) \\
\hline
\end{tabular}

Confinement, Prevent $\mathrm{H}_{2}$ explosion.

If part/component classification is different from host component/system, document basis.

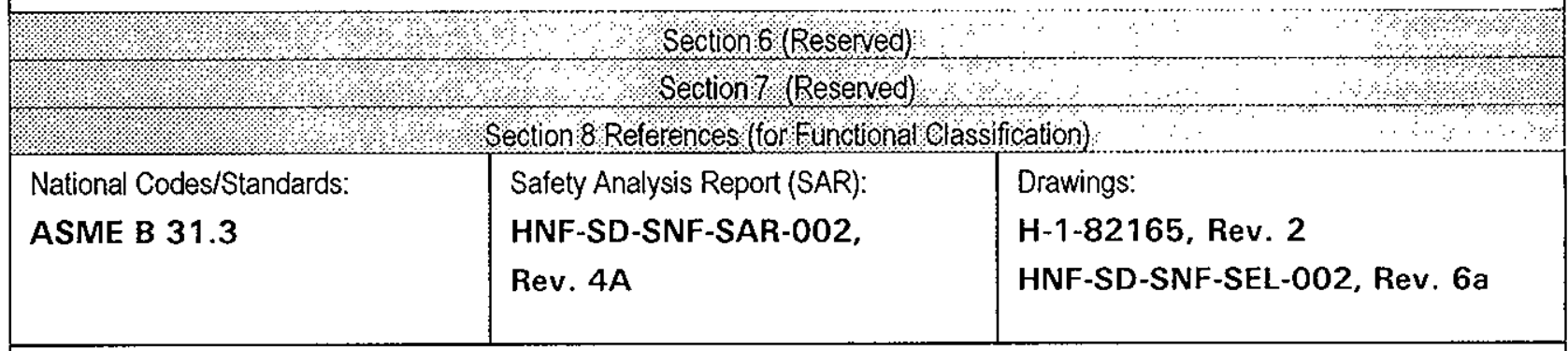

Vendor Manual/Manufacturer/Supplier Information:

Matheson Gases and Equipment, Regulators \& Gauges, P. 234.

Other: NA 
Title: MATHESON SCHe PRESSURE REGULATOR (SCHe TANK

OUTLET)

\begin{tabular}{|c|c|c|c|c|c|}
\hline \multirow{2}{*}{$\begin{array}{l}\text { Critical Characteristics Verification } \\
\text { Document: Vendor Specifications; } \\
\text { HNF-SD-SNF-SEL-002, Rev. 6a }\end{array}$} & \multicolumn{5}{|c|}{ 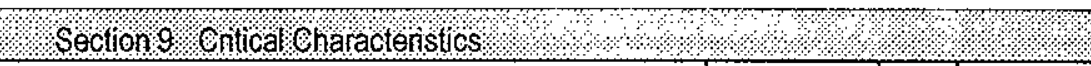 } \\
\hline & \multicolumn{2}{|l|}{ Acceptance Criteria/Tolerances } & $\begin{array}{l}\text { Acceptance } \\
\text { Melhod }\end{array}$ & ID & Function \\
\hline \multicolumn{6}{|c|}{ 1. Item Identification Critical Characteristics (necessary for reasonable assurance that the Item delivered is the Item specified) } \\
\hline Nameplate - Manufacturer & \multicolumn{2}{|c|}{ Matheson Gas Products } & 1, IN & $\mathbf{x}$ & \\
\hline $\begin{array}{l}\text { Component Number- } \\
\text { Procurement and/or Model } \\
\text { Number }\end{array}$ & \multicolumn{2}{|c|}{$\begin{array}{l}\text { 8-CGA, (Per Procurement Package W- } \\
\text { 441-P5, Rev. 2, Section G, Design Data } \\
\text { Sheet) }\end{array}$} & 1, IN & $\mathbf{x}$ & \\
\hline \multicolumn{6}{|c|}{ 2. Physical Critical Characteristics (for reasonable assurance that the Item delivered is the ltem specified) } \\
\hline Body Material & \multicolumn{2}{|c|}{ Brass (Note 4) } & $\begin{array}{l}1 . \mathrm{IN} \\
1, \mathrm{~T}\end{array}$ & $\mathbf{x}$ & \\
\hline Outlet Connection & \multicolumn{2}{|l|}{$1 / 4 "$ NPT Male } & 1, IN & $\mathbf{x}$ & \\
\hline Gauge Range & \multicolumn{2}{|l|}{$\begin{array}{l}\text { Delivery Gauge: } 0-100 \text { psig } \\
\text { Cylinder Gauge: } 0-3000 \text { psig }\end{array}$} & 1. IN & $\mathbf{x}$ & \\
\hline Weight & \multicolumn{2}{|l|}{ Nominal 7 lbs. } & 1, IN & $\mathrm{x}$ & \\
\hline \multicolumn{6}{|c|}{ 3. Performance Critical Characteristics (for reasonable assurance that the ltem will perform its intended safety function(s)) } \\
\hline Pressure Boundary & \multicolumn{2}{|c|}{$\begin{array}{l}\text { Pressure Test at } 3300 \text { psig (No Leakage- } \\
\text { No Bubbles) } \\
\text { Note } 3\end{array}$} & $1, T$ & & $x$ \\
\hline Setpoint & \multicolumn{2}{|c|}{$\begin{array}{l}\text { Maintain delivery pressure of } 25 \text { psig } \\
\text { over input pressure range of } 100 \text { to } 3000 \\
\text { psig }\end{array}$} & $1, T$ & & $\mathbf{x}$ \\
\hline Environmental & \multicolumn{2}{|l|}{ Note 1} & & & \\
\hline Seismic Condition A & Note 2 & & $1, T$ & & $x$ \\
\hline \multicolumn{3}{|l|}{ 4. Notes and Legend: } & & & \\
\hline \multicolumn{2}{|c|}{$\begin{array}{l}\text { 1. These PCVs have neoprene diaphragms. These materials are } \\
\text { not subject to degradation at } 40^{\circ} \mathrm{F} \text { and } 60 \% \mathrm{RH} \text { or } 115^{\circ} \mathrm{F} \text { and } \\
22 \% \mathrm{RH} \text { and are suitable for condition } \mathrm{B} \mathrm{Application.}\end{array}$} & & & & \\
\hline \multicolumn{2}{|c|}{$\begin{array}{l}\text { 2. Maintain critical function before and after seismic event. W- } \\
\text { 441-P5, Rev. } 2 \text {, Appendix I, page I-2, provides a seismic } \\
\text { testing plan for these components at a seismic spectra TBD. } \\
\text { Equipment that has been shaker-table tested should not be } \\
\text { installed in a plant (Ref. IEEE Standard } 344-1984 \text {, Section } 7 \text { ). } \\
\text { Consequently, the seismic test constitutes a destructive test. }\end{array}$} & & & & \\
\hline \multicolumn{2}{|c|}{$\begin{array}{l}\text { 3. Pressure test at } 110 \% \text { component internal pressure of } 3000 \\
\text { psig. }\end{array}$} & & & & \\
\hline \multicolumn{2}{|c|}{$\begin{array}{l}\text { 4. Material verification acceptance method may be by either } \\
\text { inspection or test. }\end{array}$} & & & & \\
\hline \multicolumn{4}{|c|}{ (:.:. } & & \\
\hline \multicolumn{3}{|c|}{$\begin{array}{l}\text { Approvals: } \\
\text { Designated Engineer: Com } \\
\text { Design Authority: CR mw } 6122 / 99 \\
\text { QA Engineer: TD Dawp } 6(23 / 99\end{array}$} & & & \\
\hline
\end{tabular}




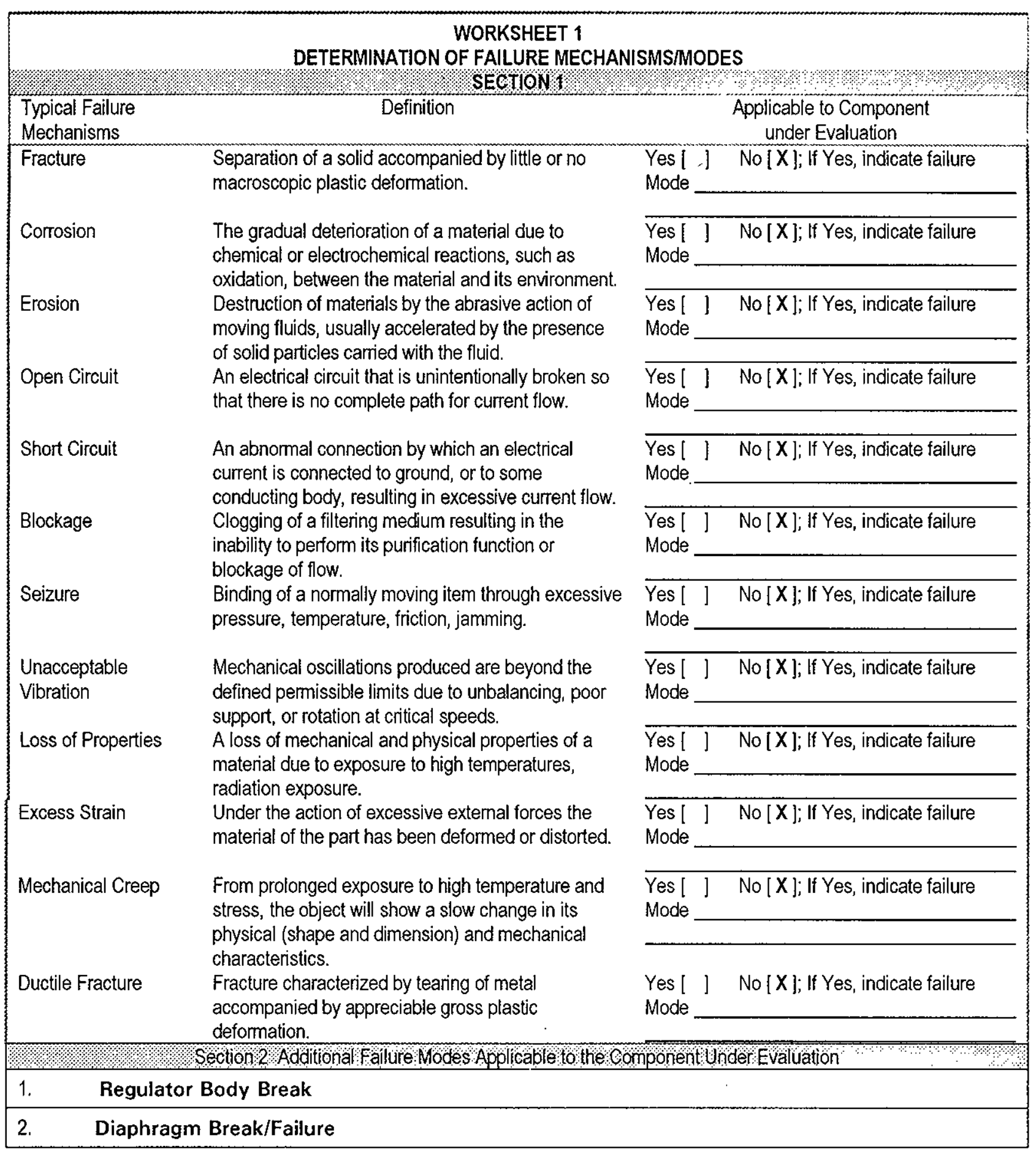


Commercial Grade Item Upgrade Dedication Form

ECN No. NA CGI No. CGI-SNF-D-13-P5-032

Title: MATHESON SCHe PRESSURE REGULATOR (SCHE TANK

OUTLET)

CHECKLIST 1

ACCEPTANCE METHOD 1

SPECIAL TEST/INSPECTION VERIFICATION

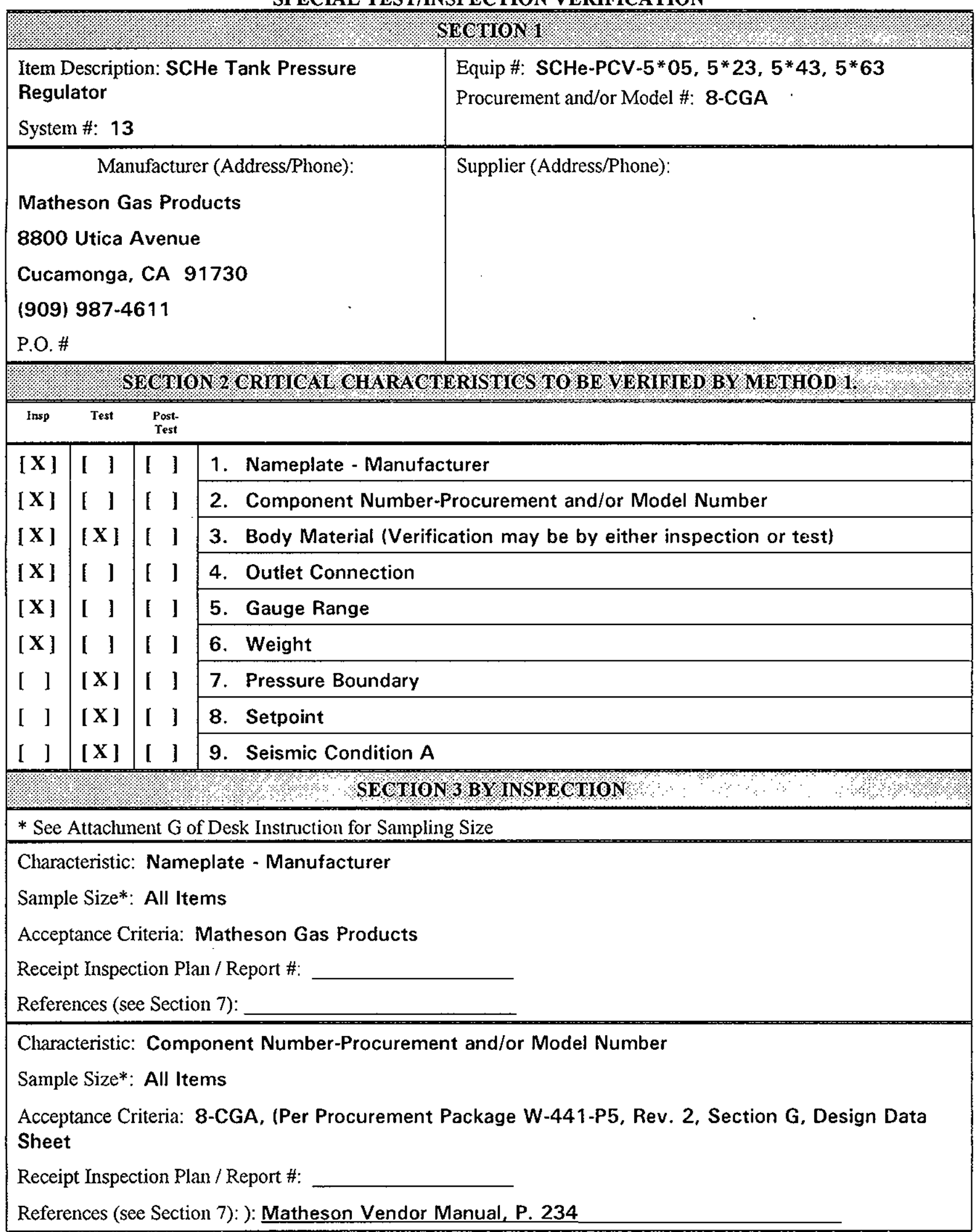




ECN No. NA CGI No. CGI-SNF-D-13-P5-032
Title: MATHESON SCHe PRESSURE REGULATOR (SCHe TANK
OUTLET)

\section{Characteristic: Outlet Connection \\ Sample Size*: All Items \\ Acceptance Criteria: $1 / 4$ " NPT Male \\ Receipt Inspection Plan / Report \#: \\ References (see Section 7):}

\section{Characteristic: Gauge Range}

Sample Size*: All Items

Acceptance Criteria: Delivery Gauge: 0-100 psig; Cylinder Gauge: 0-3000 psig

Receipt Inspection Plan / Report \#:

References (see Section 7):

Characteristic: Weight

Sample Size*: All Items

Acceptance Criteria: Nominal 7 lbs.

Receipt Inspection Plan / Report \#:

References (see Section 7):

\section{Characteristic: Body Material}

Sample Size*: Normal Sampling Size

Acceptance Criteria: Brass

Receipt Inspection Plan / Report \#:

References (see Section 7):

\section{SECTON 4 BY SPECLA TEST.}

* See Attachment G of Desk Instruction for Sampling Size

\begin{tabular}{l|l}
\hline Test To Be Performed by: & Number of Items to be Tested:
\end{tabular}

[ ] Purchaser

[ ] Supplier/Manufacturer**

Test/Inspection Location:

[ ] Other

Characteristic for Test: Pressure Boundary

Acceptance Criteria: Pressure Test at $\mathbf{3 3 0 0}$ psig (No leakage)

Sample Size*: Normal Sampling Size

Actual Test Value:

Test Plan and Report \#: References (see Section 7): 
Characteristic for Test: Setpoint

Acceptance Criteria: Maintain delivery pressure of 25 psig over input pressure range of 100 to 3000 psig. No Pressure increase above setpoint (with zero flow) for 96 hours.

Sample Size*: Normal Sampling Size

Actual Test Value:

Test Plan and Report \#: References (see Section 7):

Characteristic for Test: Seismic Condition A

Acceptance Criteria: Maintain critical function before and after seismic event

Sample Size*: W-441-P5, Rev. 2, Appendix 1, page 1-2, provides the seismic testing plan for these components. The seismic testing is conducted for one complete panel with the components assembled on the panel and tested as a complete assembly. The test seismically qualifies the entire assembly, including mountings, piping, and components. The number of components tested is dictated by the panel assembly design.

Actual Test Value:

Test Plan and Report \#: References (see Section 7):

**If Supplier/Manufacturer or Other, Refer to CGI Checklist-2 for Support Information 
Commercial Grade Item Upgrade Dedication Form SNF-3929, Rev. 2

ECN No. NA CGI No. CGI-SNF-D-13-P5-032

Title: MATHESON SCHE PRESSURE REGULATOR (SCHE TANK OUTLET)

Section 5 Test / Inspection Summary (Acceptance Method 1)

1. SUMMARY OF VERIFIED CRITICAL CHARACTERISTICS, THEIR VERIFICATION METHODS, AND RESULTS

\section{ITEM DESCRIPTION:}

\begin{tabular}{|c|c|c|c|c|c|c|c|c|c|c|c|}
\hline \multicolumn{4}{|c|}{ Critical Characteristics } & \multicolumn{8}{|c|}{ Verification Results } \\
\hline Critical Characteristics & Acceptance Criteria/Tolerances & ID & Function & $\begin{array}{l}\text { Method } \\
\text { T/IN }\end{array}$ & $\begin{array}{c}\text { Procedure } \\
\text { or } R^{\#} \#\end{array}$ & $\begin{array}{l}\text { Check- } \\
\text { list ID }\end{array}$ & $\begin{array}{l}\text { Number } \\
\text { Tested }\end{array}$ & $\begin{array}{l}\text { Number } \\
\text { Failed }\end{array}$ & $\begin{array}{l}\text { Verifying } \\
\text { Organization }\end{array}$ & Printed Name Signature & Date \\
\hline $\begin{array}{l}\text { Nameplate - } \\
\text { Manufacturer }\end{array}$ & Matheson Gas Products & $\mathbf{X}$ & & & & & & & & & \\
\hline $\begin{array}{l}\text { Component Number- } \\
\text { Procurement and/or } \\
\text { Model Number }\end{array}$ & $\begin{array}{l}\text { 8-CGA, (Per } \\
\text { Procurement Package } \\
\text { W-441-P5, Rev. 2, } \\
\text { Section G, Design Data } \\
\text { Sheet) }\end{array}$ & $\mathbf{X}$ & & & & & & & & & \\
\hline Body Material & Brass & $\mathbf{X}$ & & & & & & & & & \\
\hline Outlet Connection & $1 / 4 "$ NPT Male & $\mathbf{X}$ & & & & & & & & & \\
\hline Gauge Range & $\begin{array}{l}\text { Delivery Gauge: } 0-100 \\
\text { psig; Cylinder Gauge: } \\
0-3000 \text { psig }\end{array}$ & $\mathbf{X}$ & & & & & & & & & \\
\hline Weight & Nominal 7 lbs. & $\mathbf{X}$ & & & & & & & & & \\
\hline Pressure Boundary & $\begin{array}{l}\text { Pressure Test at } 3300 \\
\text { psig (No Leakage-No } \\
\text { Bubbles) }\end{array}$ & & $\mathbf{X}$ & & & & & & & & \\
\hline Setpoint & $\begin{array}{l}\text { Maintain delivery } \\
\text { pressure of } 25 \text { psig over } \\
\text { input pressure range of } \\
100 \text { to } 3000 \text { psig }\end{array}$ & & $\mathbf{X}$ & & & & & & & & \\
\hline Seismic Condition A & $\begin{array}{l}\text { Maintain Critical } \\
\text { Function Before and } \\
\text { After Seismic Event. }\end{array}$ & & $\mathbf{X}$ & & & & & & & . & \\
\hline
\end{tabular}


Commercial Grade Item Upgrade Dedication Form

Title: MATHESON SCHe PRESSURE REGULATOR (SCHE TANK

OUTLET)

Secton $6 \%$ ontacts/Phon o Numbers

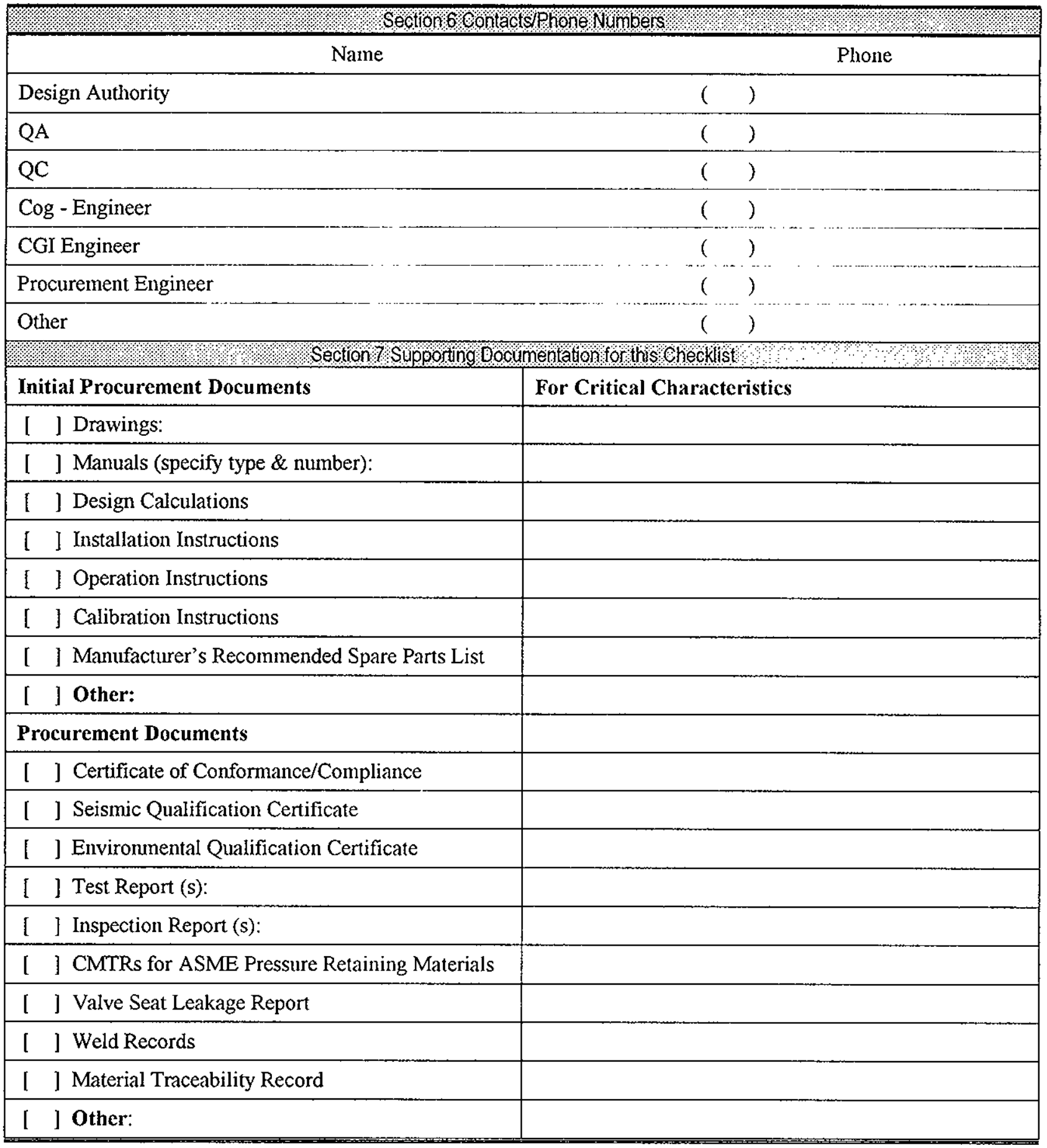

